\title{
Dirichlet Forms Constructed from Annihilation Operators on Bernoulli Functionals
}

\author{
Caishi Wang and Beiping Wang \\ School of Mathematics and Statistics, Northwest Normal University, Lanzhou, Gansu 730070, China \\ Correspondence should be addressed to Caishi Wang; cswangnwnu@163.com
}

Received 30 September 2016; Accepted 17 January 2017; Published 8 February 2017

Academic Editor: Antonio Scarfone

Copyright (C) 2017 Caishi Wang and Beiping Wang. This is an open access article distributed under the Creative Commons Attribution License, which permits unrestricted use, distribution, and reproduction in any medium, provided the original work is properly cited.

\begin{abstract}
The annihilation operators on Bernoulli functionals (Bernoulli annihilators, for short) and their adjoint operators satisfy a canonical anticommutation relation (CAR) in equal-time. As a mathematical structure, Dirichlet forms play an important role in many fields in mathematical physics. In this paper, we apply the Bernoulli annihilators to constructing Dirichlet forms on Bernoulli functionals. Let $w$ be a nonnegative function on $\mathbb{N}$. By using the Bernoulli annihilators, we first define in a dense subspace of $L^{2}$-space of Bernoulli functionals a positive, symmetric, bilinear form $\mathscr{E}_{w}$ associated with $w$. And then we prove that $\mathscr{E}_{w}$ is closed and has the contraction property; hence, it is a Dirichlet form. Finally, we consider an interesting semigroup of operators associated with $w$ on $L^{2}$-space of Bernoulli functionals, which we call the $w$-Ornstein-Uhlenbeck semigroup, and, by using the Dirichlet form, $\mathscr{E}_{w}$ we show that the $w$-Ornstein-Uhlenbeck semigroup is a Markov semigroup.
\end{abstract}

\section{Introduction}

The annihilation operators on Bernoulli functionals (Bernoulli annihilators, for short) admit much good operation properties with physical meanings. For example, they together with their adjoint operators satisfy a canonical anticommutation relation (CAR) in equal-time [1]. In recent years, these operators have begun to find applications in developing a discrete-time stochastic calculus in infinite dimensions. Privault [2] used the Bernoulli annihilators to define the gradients for Bernoulli functionals in 2008. In 2010, Nourdin et al. [3] investigated normal approximation of Rademacher functionals (a special case of Bernoulli functionals) with the help of the Bernoulli annihilators. Recently [4], it has been shown that a wide class of quantum Markov semigroups can be constructed from the Bernoulli annihilators and their adjoint operators. As is known, quantum Markov semigroups are quantum analogues of the classical Markov semigroups in probability theory, which provide a mathematical model for describing the irreversible evolution of quantum systems interacting with the environment (see, e.g., $[5-7])$.

A Dirichlet form [8] in an $L^{2}$-space is a closed, positive, symmetric, densely defined bilinear form that has the contraction property (also known as the Markov property). As a mathematical structure, Dirichlet forms have close connections with many objects in probability theory, quantum mechanics, and quantum field theory (see [8] and references therein). One typical example in this respect is that, under some mild conditions, a Dirichlet form determines a Markov process through the Markov semigroup associated with it [9]. Thus, Dirichlet forms play an important role in construction of Markov processes [9]. The classical Dirichlet forms [9, 10] are usually defined in the $L^{2}$-spaces of functions on locally compact metric spaces. In the past three decades, however, Dirichlet forms on noncommutative and even infinite dimensional settings have also appeared successively. For example, Albeverio and Hoegh-Krohn [11] constructed Dirichlet forms on $C^{*}$-algebras, while Hida et al. [12] gave a Dirichlet form on white noise functionals by using Hida's differential operators. 
In this paper, motivated by the work of Hida et al. [12], we would like to apply the Bernoulli annihilators to constructing Dirichlet forms on Bernoulli functionals. Our main work is as follows.

Let $\left\{\partial_{k}\right\}_{k \geq 0}$ be the Bernoulli annihilators. For a nonnegative function $w$ on $\mathbb{N}$, we first define in the $L^{2}$-space of Bernoulli functionals a positive, symmetric, densely defined bilinear form $\mathscr{E}_{w}$ in the following manner:

$$
\mathscr{E}_{w}(\xi, \eta)=\sum_{k=0}^{\infty} w(k)\left\langle\partial_{k} \xi, \partial_{k} \eta\right\rangle
$$

where $\langle\cdot, \cdot\rangle$ denotes the inner product in the $L^{2}$-space of Bernoulli functionals. We then prove that $\mathscr{E}_{w}$ is closed and has the contraction property; hence, it is a Dirichlet form. We also obtain an operator representation of $\mathscr{E}_{w}$. Finally, we consider an interesting semigroup of operators associated with $w$ on the $L^{2}$-space of Bernoulli functionals, which we call the $w$-Ornstein-Uhlenbeck semigroup, and, by using the Dirichlet form $\mathscr{E}_{w}$, we show that the $w$-Ornstein-Uhlenbeck semigroup is a Markov semigroup.

We mention that the whole family of the annihilation and creation operators acting on Bernoulli functionals is interpreted as a type of quantum Bernoulli noises $[1,13]$. As is seen, however, we only make use of the annihilation operators in the construction of our Dirichlet form. Thus, our work here does not mean that one can construct a Dirichlet form from quantum Bernoulli noises. In fact, from a physical point of view, any Dirichlet forms cannot be constructed from any quantum Bernoulli noises.

Notation and Conventions. Throughout, $\mathbb{N}$ always denotes the set of all nonnegative integers. We denote by $\Gamma$ the finite power set of $\mathbb{N}$; namely,

$$
\Gamma=\{\sigma \mid \sigma \subset \mathbb{N}, \# \sigma<\infty\},
$$

where $\#(\sigma)$ means the cardinality of $\sigma$ as a set. Unless otherwise stated, letters like $j, k$, and $n$ stand for nonnegative integers, namely, elements of $\mathbb{N}$.

\section{Bernoulli Annihilators}

In this section, we briefly recall some necessary notions and facts about Bernoulli functionals and the annihilation operators on them. For details, we refer to [1].

Let $\Omega=\{-1,1\}^{\mathbb{N}}$ be the set of all mappings $\omega: \mathbb{N} \mapsto$ $\{-1,1\}$ and $\left(\zeta_{n}\right)_{n \geq 0}$ the sequence of canonical projections on $\Omega$ given by

$$
\zeta_{n}(\omega)=\omega(n), \quad \omega \in \Omega
$$

Denote by $\mathscr{F}$ the $\sigma$-field on $\Omega$ generated by the sequence $\left(\zeta_{n}\right)_{n \geq 0}$. Let $\left(p_{n}\right)_{n \geq 0}$ be a given sequence of positive numbers with the property that $0<p_{n}<1$ for all $n \geq 0$. It is known [2] that there exists a unique probability measure $\mathbb{P}$ on $\mathscr{F}$ such that

$$
\begin{aligned}
\mathbb{P} \circ & \left(\zeta_{n_{1}}, \zeta_{n_{2}}, \ldots, \zeta_{n_{k}}\right)^{-1}\left\{\left(\epsilon_{1}, \epsilon_{2}, \ldots, \epsilon_{k}\right)\right\} \\
= & \prod_{j=1}^{k} p_{j}^{\left(1+\epsilon_{j}\right) / 2}\left(1-p_{j}\right)^{\left(1-\epsilon_{j}\right) / 2} .
\end{aligned}
$$

For $n_{j} \in \mathbb{N}, \epsilon_{j} \in\{-1,1\}(1 \leq j \leq k)$ with $n_{i} \neq n_{j}$ when $i \neq j$ and $k \in \mathbb{N}$ with $k \geq 1$. Thus we have a probability measure space $(\Omega, \mathscr{F}, \mathbb{P})$.

Let $Z=\left(Z_{n}\right)_{n \geq 0}$ be the sequence of random variables on $(\Omega, \mathscr{F}, \mathbb{P})$ defined by

$$
Z_{n}=\frac{\zeta_{n}+q_{n}-p_{n}}{2 \sqrt{p_{n} q_{n}}}, \quad n \geq 0,
$$

where $q_{n}=1-p_{n}$. Clearly $Z=\left(Z_{n}\right)_{n \geq 0}$ is an independent sequence of random variables on $(\Omega, \mathscr{F}, \mathbb{P})$, and, for each $n \geq$ $0, Z_{n}$ has a distribution:

$$
\begin{gathered}
\mathbb{P}\left\{Z_{n}=\theta_{n}\right\}=p_{n}, \\
\mathbb{P}\left\{Z_{n}=-\frac{1}{\theta_{n}}\right\}=q_{n}, \\
n \geq 0
\end{gathered}
$$

with $\theta_{n}=\sqrt{q_{n} / p_{n}}$. To be convenient, we set $\mathscr{F}_{-1}=\{\emptyset, \Omega\}$ and

$$
\mathscr{F}_{n}=\sigma\left(Z_{k} ; 0 \leq k \leq n\right),
$$

the $\sigma$-field generated by $\left(Z_{k}\right)_{0 \leq k \leq n}$ for $n \geq 0$. By convention, $\mathbb{E}$ will denote the expectation with respect to $\mathbb{P}$.

Let $L^{2}(\Omega)$ be the space of square integrable random variables on $(\Omega, \mathscr{F}, \mathbb{P})$, namely,

$$
L^{2}(\Omega)=L^{2}(\Omega, \mathscr{F}, \mathbb{P}) .
$$

We denote by $\langle\cdot, \cdot\rangle$ the usual inner product of the space $L^{2}(\Omega)$ and by $\|\cdot\|$ the corresponding norm. It is known [2] that $Z$ has the chaotic representation property. Thus $L^{2}(\Omega)$ has $\left\{Z_{\sigma} \mid\right.$ $\sigma \in \Gamma\}$ as its orthonormal basis, where $Z_{\emptyset}=1$ and

$$
Z_{\sigma}=\prod_{j \in \sigma} Z_{j}, \quad \sigma \in \Gamma, \sigma \neq \emptyset,
$$

which shows that $L^{2}(\Omega)$ is an infinite dimensional real Hilbert space.

Remark 1. It is easy to see that $\mathscr{F}=\left(Z_{n} ; n \geq 0\right)$. Thus $\mathscr{F}$ measurable functions on $\Omega$ are usually known as functionals of $Z$ or Bernoulli functionals simply. In particular, functions in $L^{2}(\Omega)$ are called square integrable Bernoulli functionals.

Lemma 2 (see [1]). For each $k \in \mathbb{N}$, there exists a bounded operator $\partial_{k}$ on $L^{2}(\Omega)$ such that

$$
\partial_{k} Z_{\sigma}=\mathbf{1}_{\sigma}(k) Z_{\sigma \backslash k}, \quad \sigma \in \Gamma,
$$

where $\sigma \backslash k=\sigma \backslash\{k\}$ and $\mathbf{1}_{\sigma}(k)$ is the indicator of $\sigma$ as a subset of $\mathbb{N}$. 
Lemma 3 (see [1]). Let $k \in \mathbb{N}$. Then $\partial_{k}^{*}$, the adjoint of operator $\partial_{k}$, has following property:

$$
\partial_{k}^{*} Z_{\sigma}=\left[1-\mathbf{1}_{\sigma}(k)\right] Z_{\sigma \cup k}, \quad \sigma \in \Gamma,
$$

where $\sigma \cup k=\sigma \cup\{k\}$.

The operator $\partial_{k}$ and its adjoint $\partial_{k}^{*}$ are referred to as the annihilation operator and creation operator at site $k$, respectively. The next lemma shows that these operators satisfy a canonical anticommutation relations (CAR) in equal-time.

Lemma 4 (see [1]). Let $k, l \in \mathbb{N}$. Then it holds true that

$$
\begin{aligned}
\partial_{k} \partial_{l} & =\partial_{l} \partial_{k}, \\
\partial_{k}^{*} \partial_{l}^{*} & =\partial_{l}^{*} \partial_{k}^{*}, \\
\partial_{k}^{*} \partial_{l} & =\partial_{l} \partial_{k}^{*}, \quad(k \neq l), \\
\partial_{k} \partial_{k} & =\partial_{k}^{*} \partial_{k}^{*}=0, \\
\partial_{k} \partial_{k}^{*}+\partial_{k}^{*} \partial_{k} & =I,
\end{aligned}
$$

where $I$ is the identity operator on $L^{2}(\Omega)$.

\section{Forms Constructed from Bernoulli Annihilators}

In the present section, we show how to use the annihilation operators $\left\{\partial_{k}\right\}_{k \geq 0}$ to construct a closed, positive, symmetric, and densely defined bilinear form in $L^{2}(\Omega)$.

We first prove a convergence result concerning $\left\{\partial_{k}\right\}_{k \geq 0}$, which will play a key role in our later discussions.

For a nonnegative function $w: \mathbb{N} \rightarrow \mathbb{R}$, we define the $w$-counting measure $\#_{w}(\cdot)$ as

$$
\#_{w}(\sigma)=\sum_{j \in \sigma} w(j), \quad \sigma \in \Gamma,
$$

where $\#_{w}(\sigma)=0$ if $\sigma=\emptyset$. Clearly, $0 \leq \#_{w}(\sigma)<\infty$ for all $\sigma \in \Gamma$.

Theorem 5. Let $w: \mathbb{N} \rightarrow \mathbb{R}$ be a nonnegative function. Define $\mathrm{D}_{w}$ as the linear subspace of $L^{2}(\Omega)$ given by

$$
\mathrm{D}_{w}=\left\{\left.\xi \in L^{2}(\Omega)\left|\sum_{\sigma \in \Gamma} \#_{w}(\sigma)\right|\left\langle Z_{\sigma}, \xi\right\rangle\right|^{2}<\infty\right\} .
$$

Then, $\mathrm{D}_{w}$ is a dense linear subspace of $L^{2}(\Omega)$, and, moreover, for all $\xi, \eta \in \mathrm{D}_{w}$, the following series, is absolutely convergent:

$$
\sum_{k=0}^{\infty} w(k)\left\langle\partial_{k} \xi, \partial_{k} \eta\right\rangle
$$

Proof. Clearly, $\mathrm{D}_{w}$ is a linear subspace of $L^{2}(\Omega)$. On the other hand, for each $\tau \in \Gamma$, in view of the fact that $\#_{w}(\tau)<\infty$, we have

$$
\sum_{\sigma \in \Gamma} \#_{w}(\sigma)\left|\left\langle Z_{\sigma}, Z_{\tau}\right\rangle\right|^{2}=\#_{w}(\tau)<\infty
$$

Thus, $\left\{Z_{\tau} \mid \tau \in \Gamma\right\} \subset \mathrm{D}_{w}$, which implies that $\mathrm{D}_{w}$ is dense in $L^{2}(\Omega)$.

Now, let $\xi, \eta \in \mathrm{D}_{w}$. Then, for each $k \geq 0$, it follows from Lemma 2 as well as the expansion $\xi=\sum_{\sigma \in \Gamma}\left\langle Z_{\sigma}, \xi\right\rangle Z_{\sigma}$ that

$$
\partial_{k} \xi=\sum_{\sigma \in \Gamma} \mathbf{1}_{\sigma}(k)\left\langle Z_{\sigma}, \xi\right\rangle Z_{\sigma \backslash k}
$$

Thus, by a direct calculation, we have

$$
\begin{aligned}
\sum_{k=0}^{\infty} w(k)\left\|\partial_{k} \xi\right\|^{2} & =\sum_{k=0}^{\infty} \sum_{\sigma \in \Gamma} \mathbf{1}_{\sigma}(k) w(k)\left|\left\langle Z_{\sigma}, \xi\right\rangle\right|^{2} \\
& =\sum_{\sigma \in \Gamma} \#_{w}(\sigma)\left|\left\langle Z_{\sigma}, \xi\right\rangle\right|^{2}<\infty .
\end{aligned}
$$

With the same argument, we have

$$
\sum_{k=0}^{\infty} w(k)\left\|\partial_{k} \eta\right\|^{2}<\infty
$$

It then follows from these two equalities that

$$
\begin{aligned}
\sum_{k=0}^{\infty} & w(k)\left|\left\langle\partial_{k} \xi, \partial_{k} \eta\right\rangle\right| \\
& \leq\left[\sum_{k=0}^{\infty} w(k)\left\|\partial_{k} \xi\right\|^{2}\right]^{1 / 2}\left[\sum_{k=0}^{\infty} w(k)\left\|\partial_{k} \eta\right\|^{2}\right]^{1 / 2} \\
& <\infty
\end{aligned}
$$

which implies that the series $\sum_{k=0}^{\infty} w(k)\left\langle\partial_{k} \xi, \partial_{k} \eta\right\rangle$ is absolutely convergent.

In view of the above theorem, we come naturally to the next definition, which introduces our main object of study.

Definition 6. For a nonnegative function $w: \mathbb{N} \rightarrow \mathbb{R}$, we define $\mathscr{E}_{w}$ as

$$
\mathscr{E}_{w}(\xi, \eta)=\sum_{k=0}^{\infty} w(k)\left\langle\partial_{k} \xi, \partial_{k} \eta\right\rangle, \quad \xi, \eta \in \mathrm{D}_{w},
$$

and call $\left(\mathscr{E}_{w}, \mathrm{D}_{w}\right)$ the $w$-energy form.

It is easy to see that $\left(\mathscr{E}_{w}, \mathrm{D}_{w}\right)$ is a positive, symmetric, and densely defined bilinear form in $L^{2}(\Omega)$. Note that if we take $w(k) \equiv 1$, then we get

$$
\mathscr{E}_{w}(\xi, \xi)=\int_{\Omega}\|\nabla \xi(\omega)\|_{l^{2}(\mathbb{N})}^{2} d \mathbb{P}(\omega), \quad \xi \in D_{w},
$$

where $\nabla$ denotes the gradient operator, which is defined on $\mathrm{D}_{w}$ and valued in $L^{2}(\Omega \times \mathbb{N})$, the space of jointly square integrable stochastic processes on $(\Omega, \mathscr{F}, \mathbb{P})$ (see [14] for details). This justifies the name of $\left(\mathscr{E}_{w}, \mathrm{D}_{w}\right)$.

In order to examine basic properties of the $w$-energy form $\left(\mathscr{E}_{w}, \mathrm{D}_{w}\right)$, we introduce another bilinear form $\widehat{\mathscr{E}}_{w}$ on $\mathrm{D}_{w}$ as

$$
\widehat{\mathscr{E}}_{w}(\xi, \eta)=\mathscr{E}_{w}(\xi, \eta)+\langle\xi, \eta\rangle, \quad \xi, \eta \in \mathrm{D}_{w},
$$


where $\langle\cdot, \cdot\rangle$ is the inner product of $L^{2}(\Omega)$. It is then easy to see that $\widehat{\mathscr{E}}_{w}$ is again an inner product on $D_{w}$. We denote by $\|\cdot\|_{\widehat{\mathscr{b}}_{w}}$ the norm induced by $\widehat{\mathscr{E}}_{w}$. A direct calculation gives that

$$
\|\xi\|_{\widehat{\mathscr{O}}_{w}}^{2}=\sum_{\sigma \in \Gamma}\left[\#_{w}(\sigma)+1\right]\left|\left\langle Z_{\sigma}, \xi\right\rangle\right|^{2}, \quad \xi \in \mathrm{D}_{w}
$$

where $\#_{w}(\cdot)$ is the $w$-counting measure as defined by (13).

Theorem 7. Let $w: \mathbb{N} \rightarrow \mathbb{R}$ be a nonnegative function. Then the w-energy form $\left(\mathscr{E}_{w}, \mathrm{D}_{w}\right)$ is closed; namely, $\left(\mathrm{D}_{w}, \widehat{\mathscr{E}}_{w}\right)$ is a Hilbert space.

Proof. We need only to show that $\mathrm{D}_{w}$ is complete with respect to norm $\|\cdot\|_{\widehat{\mathscr{C}}_{w}}$.

Let $\left\{\xi_{n}\right\}_{n \geq 1} \subset D_{w}$ be a Cauchy sequence with respect to norm $\|\cdot\|_{\widehat{\mathscr{O}}_{w}}$. Then it is also a Cauchy sequence with respect to norm $\|\cdot\|$ since $\|\cdot\| \leq\|\cdot\|_{\widehat{\mathscr{b}}_{w}}$. Thus there exists $\xi \in L^{2}(\Omega)$ such that $\left\|\xi_{n}-\xi\right\| \rightarrow 0$ as $n \rightarrow \infty$. Now we show that $\xi \in \mathrm{D}_{w}$ and $\left\|\xi_{n}-\xi\right\|_{\widehat{\mathscr{\delta}}_{w}} \rightarrow 0$ as $n \rightarrow \infty$.

Let $\epsilon>0$. Then, by the property that $\left\{\xi_{n}\right\}_{n \geq 1}$ is a Cauchy sequence with respect to $\|\cdot\|_{\widehat{\mathscr{E}}_{w}}$, we know that there exists a positive integer $K \geq 1$ such that

$$
\sum_{\sigma \in \Gamma}\left[\#_{w}(\sigma)+1\right]\left|\left\langle Z_{\sigma}, \xi_{m}-\xi_{n}\right\rangle\right|^{2}=\left\|\xi_{m}-\xi_{n}\right\|_{\mathscr{\mathscr { E }}_{w}}^{2}<\epsilon^{2},
$$

$$
\forall m, n>K \text {. }
$$

For all $n>K$, since

$$
\begin{aligned}
& \lim _{m \rightarrow \infty}\left[\#_{w}(\sigma)+1\right]\left|\left\langle Z_{\sigma}, \xi_{m}-\xi_{n}\right\rangle\right|^{2} \\
& \quad=\left[\#_{w}(\sigma)+1\right]\left|\left\langle Z_{\sigma}, \xi-\xi_{n}\right\rangle\right|^{2}, \quad \forall \sigma \in \Gamma,
\end{aligned}
$$

it follows by applying the well-known Fatou's Lemma [15] to (25) that

$$
\sum_{\sigma \in \Gamma}\left[\#_{w}(\sigma)+1\right]\left|\left\langle Z_{\sigma}, \xi-\xi_{n}\right\rangle\right|^{2} \leq \epsilon^{2},
$$

which implies that $\xi-\xi_{n} \in \mathrm{D}_{w}$; hence $\xi \in \mathrm{D}_{w}$ and

$$
\begin{aligned}
\left\|\xi-\xi_{n}\right\|_{\widehat{\mathscr{E}}_{w}} & =\left\{\sum_{\sigma \in \Gamma}\left[\#_{w}(\sigma)+1\right]\left|\left\langle Z_{\sigma}, \xi-\xi_{n}\right\rangle\right|^{2}\right\}^{1 / 2} \\
& \leq \epsilon .
\end{aligned}
$$

Therefore, $\left\|\xi_{n}-\xi\right\|_{\widehat{\mathscr{\delta}}_{w}} \rightarrow 0$ as $n \rightarrow \infty$; namely, $\left\{\xi_{n}\right\}$ converges in $\mathrm{D}_{w}$ with respect to norm $\|\cdot\|_{\widehat{\mathscr{b}}_{w}}$.

\section{Contraction Property}

Let $w: \mathbb{N} \rightarrow \mathbb{R}$ be a given nonnegative function. In this section, we further prove that the $w$-energy form $\left(\mathscr{E}_{w}, \mathrm{D}_{w}\right)$ has the contraction property; hence, it is a Dirichlet form.

We first make some necessary preparations. Let $S=$ $\operatorname{Span}\left\{Z_{\sigma} \mid \sigma \in \Gamma\right\}$, the linear subspace of $L^{2}(\Omega)$ spanned by the system $\left\{Z_{\sigma} \mid \sigma \in \Gamma\right\}$, which is obviously a dense linear subspace of $L^{2}(\Omega)$. For $n \geq 0$, we denote by $S_{n}$ the linear subspace of all $\mathscr{F}_{n}$-measurable random variables in $L^{2}(\Omega)$. It can be verified that $S_{n}$ is a $2^{n+1}$-dimensional closed subspace of $L^{2}(\Omega)$ and has an orthonormal basis $\left\{Z_{\sigma} \mid \sigma \in \Gamma_{n}\right\}$, where $\Gamma_{n]}=\{\sigma \in \Gamma \mid \max \sigma \leq n\}$. And, moreover, these subspaces have the following relation:

$$
\bigcup_{n=0}^{\infty} \mathrm{S}_{n}=\mathrm{S} .
$$

Recall that elements of $\Omega$ are mappings $\omega: \mathbb{N} \rightarrow\{-1,1\}$. For $k \geq 0$ and $\omega \in \Omega$, we can naturally define two mappings $\omega_{k}^{+}, \omega_{k}^{-}: \mathbb{N} \rightarrow\{-1,1\}$ as

$$
\begin{aligned}
& \omega_{k}^{+}(n)= \begin{cases}1, & n=k ; \\
\omega(n), & n \neq k,\end{cases} \\
& \omega_{k}^{-}(n)= \begin{cases}-1, & n=k ; \\
\omega(n), & n \neq k,\end{cases}
\end{aligned}
$$

which, of course, remain elements of $\Omega$.

The following proposition is a slight variant of a result given in [2], which shows that the annihilation operator $\partial_{k}$ acts just like a difference operator.

Lemma 8 (see [2]). Let $k \geq 0$ and $\xi \in \mathrm{S}$. Then,

$$
\partial_{k} \xi(\omega)=\sqrt{p_{k} q_{k}}\left[\xi\left(\omega_{k}^{+}\right)-\xi\left(\omega_{k}^{-}\right)\right], \quad \omega \in \Omega .
$$

Proof. We need only to show that (31) holds for each $\xi=Z_{\sigma}$ with $\sigma \in \Gamma$. Let $\sigma \in \Gamma$. Then, it follows from (3), (5), and (30) that

$$
\begin{aligned}
& Z_{j}(\omega)=Z_{j}\left(\omega_{k}^{+}\right)=Z_{j}\left(\omega_{k}^{-}\right), \quad \omega \in \Omega, j \neq k, \\
& Z_{k}\left(\omega_{k}^{+}\right)=\sqrt{\frac{q_{k}}{p_{k}}}, \\
& Z_{k}\left(\omega_{k}^{-}\right)=-\sqrt{\frac{p_{k}}{q_{k}}} .
\end{aligned}
$$

Thus, in case of $k \notin \sigma, Z_{\sigma}(\omega)=Z_{\sigma}\left(\omega_{k}^{+}\right)=Z_{\sigma}\left(\omega_{k}^{-}\right)$for $\omega \in \Omega$, which implies

$$
\sqrt{p_{k} q_{k}}\left[Z_{\sigma}\left(\omega_{k}^{+}\right)-Z_{\sigma}\left(\omega_{k}^{-}\right)\right]=0, \quad \omega \in \Omega,
$$

which, together with the formula $\partial_{k} Z_{\sigma}=\mathbf{1}_{\sigma}(k) Z_{\sigma \backslash k}$, gives

$$
\partial_{k} Z_{\sigma}(\omega)=\sqrt{p_{k} q_{k}}\left[Z_{\sigma}\left(\omega_{k}^{+}\right)-Z_{\sigma}\left(\omega_{k}^{-}\right)\right], \quad \omega \in \Omega .
$$

Now, if $k \in \sigma$, then $Z_{\sigma \backslash k}(\omega)=Z_{\sigma \backslash k}\left(\omega_{k}^{+}\right)=Z_{\sigma \backslash k}\left(\omega_{k}^{-}\right)$for $\omega \epsilon$ $\Omega$, which together with (33) yields

$$
\begin{aligned}
& Z_{\sigma \backslash k}(\omega) \\
& \quad=\sqrt{p_{k} q_{k}}\left[Z_{\sigma \backslash k}\left(\omega_{k}^{+}\right) \sqrt{\frac{q_{k}}{p_{k}}}+Z_{\sigma \backslash k}\left(\omega_{k}^{-}\right) \sqrt{\frac{p_{k}}{q_{k}}}\right] \\
& \quad=\sqrt{p_{k} q_{k}}\left[Z_{\sigma \backslash k}\left(\omega_{k}^{+}\right) Z_{k}\left(\omega_{k}^{+}\right)-Z_{\sigma \backslash k}\left(\omega_{k}^{-}\right) Z_{k}\left(\omega_{k}^{-}\right)\right] \\
& =\sqrt{p_{k} q_{k}}\left[Z_{\sigma}\left(\omega_{k}^{+}\right)-Z_{\sigma}\left(\omega_{k}^{-}\right)\right], \quad \omega \in \Omega,
\end{aligned}
$$


which, together with the formula $\partial_{k} Z_{\sigma}=\mathbf{1}_{\sigma}(k) Z_{\sigma \backslash k}$, still leads to

$$
\partial_{k} Z_{\sigma}(\omega)=\sqrt{p_{k} q_{k}}\left[Z_{\sigma}\left(\omega_{k}^{+}\right)-Z_{\sigma}\left(\omega_{k}^{-}\right)\right], \quad \omega \in \Omega
$$

This completes the proof.

Definition 9. A contraction function is a function $C: \mathbb{R} \rightarrow \mathbb{R}$ with $C(0)=0$, and $|C(s)-C(t)| \leq|s-t|$ for all $s, t \in \mathbb{R}$.

As usual, we use $C \circ \xi$ to mean the composition of a contraction function $C$ and a random variable $\xi$. The next theorem then shows that $S$ is invariant under the action of contraction functions.

Theorem 10. Let $C$ be a contraction function. Then $C \circ \xi \in \mathrm{S}$ for all $\xi \in \mathrm{S}$.

Proof. Let $\xi \in \mathrm{S}$. Then there exists some $n \geq 0$ such that $\xi \in$ $\mathrm{S}_{n}$, which implies $C \circ \xi \in \mathrm{S}_{n}$ since $C$ is a continuous function. Thus $C \circ \xi \in S$.

Theorem 11. Let $\xi \in L^{2}(\Omega)$ and $C$ be a contraction function. Then, for all $k \geq 0$, it holds that

$$
\left\|\partial_{k}(C \circ \xi)\right\| \leq\left\|\partial_{k} \xi\right\|
$$

Proof. Let $k \geq 0$. Then, we can take a sequence $\left(\xi_{n}\right)_{n \geq 1}$ in $S$ such that $\left\|\xi_{n}-\xi\right\| \rightarrow 0$ as $n \rightarrow \infty$. It follows from Theorem 10 that $\left\{C \circ \xi_{n}\right\}_{n \geq 1} \subset \mathrm{S}$. Thus, for each $n \geq 1$, by using Lemma 8 and the contraction property of $C$, we have

$$
\begin{aligned}
\left|\partial_{k}\left(C \circ \xi_{n}\right)(\omega)\right| & =\sqrt{p_{k} q_{k}}\left|C \circ \xi_{n}\left(\omega_{k}^{+}\right)-C \circ \xi_{n}\left(\omega_{k}^{-}\right)\right| \\
& \leq \sqrt{p_{k} q_{k}}\left|\xi_{n}\left(\omega_{k}^{+}\right)-\xi_{n}\left(\omega_{k}^{-}\right)\right| \\
& =\left|\partial_{k} \xi_{n}(\omega)\right|
\end{aligned}
$$

with $\omega \in \Omega$, which implies

$$
\left\|\partial_{k}\left(C \circ \xi_{n}\right)\right\| \leq\left\|\partial_{k} \xi_{n}\right\|, \quad n \geq 1 .
$$

It is easy to see that $\left\|C \circ \xi_{n}-C \circ \xi\right\| \rightarrow 0$ as $n \rightarrow \infty$. Thus, by letting $n \rightarrow \infty$ in the above inequality, we finally come to (38).

Theorem 12. The w-energy form $\left(\mathscr{E}_{w}, \mathrm{D}_{w}\right)$ has the contraction property; namely, for all contraction function $C: \mathbb{R} \rightarrow \mathbb{R}$ and all $\xi \in \mathrm{D}_{w}$, it holds that $C \circ \xi \in \mathrm{D}_{w}$ and

$$
\mathscr{E}_{w}(C \circ \xi, C \circ \xi) \leq \mathscr{E}_{w}(\xi, \xi)
$$

Proof. Let $\xi \in \mathrm{D}_{w}$ and $C: \mathbb{R} \rightarrow \mathbb{R}$ be a contraction function. Then, by Theorem 11, we have

$$
\begin{gathered}
\sum_{\sigma \in \Gamma} \#_{w}(\sigma)\left|\left\langle Z_{\sigma}, C \circ \xi\right\rangle\right|^{2}=\sum_{k=0}^{\infty} w(k)\left\|\partial_{k}(C \circ \xi)\right\|^{2} \\
\leq \sum_{k=0}^{\infty} w(k)\left\|\partial_{k} \xi\right\|^{2}=\sum_{\sigma \in \Gamma} \#_{w}(\sigma)\left|\left\langle Z_{\sigma}, \xi\right\rangle\right|^{2},
\end{gathered}
$$

which, together with the assumption $\xi \in D_{w}$, implies $C \circ \xi \in$ $\mathrm{D}_{w}$. It then follows from the definition of $\mathscr{E}_{w}$ that

$$
\begin{aligned}
\mathscr{E}_{w}(C \circ \xi, C \circ \xi) & =\sum_{k=0}^{\infty} w(k)\left\|\partial_{k}(C \circ \xi)\right\|^{2} \\
& \leq \sum_{k=0}^{\infty} w(k)\left\|\partial_{k} \xi\right\|^{2}=\mathscr{E}_{w}(\xi, \xi) .
\end{aligned}
$$

In the literature, a closed, positive, symmetric, densely defined bilinear form in an $L^{2}$-space is called a Dirichlet form if it additionally has the contraction property (see, e.g., $[8,9,12])$. Summing up our discussions above, we actually arrive at the next important conclusion.

Corollary 13. The w-energy form $\left(\mathscr{E}_{w}, \mathrm{D}_{w}\right)$ is a Dirichlet form in $L^{2}(\Omega)$.

\section{Application}

Markov semigroups are semigroups of contraction operators that are closely related to Markov processes in probability theory. In the final section, we consider an interesting class of semigroups of contraction operators on $L^{2}(\Omega)$. As an application of our results in the previous sections, we will prove that this class of semigroups are actually Markov ones.

Let $w: \mathbb{N} \rightarrow \mathbb{R}$ be a nonnegative function. For each $t \geq 0$, one can define a contraction operator $P_{t}^{w}$ on $L^{2}(\Omega)$ as

$$
P_{t}^{w} \xi=\sum_{\sigma \in \Gamma} e^{-t \#_{w}(\sigma)}\left\langle Z_{\sigma}, \xi\right\rangle Z_{\sigma}, \quad \xi \in L^{2}(\Omega),
$$

where $\#_{w}(\cdot)$ is the $w$-counting measure defined by (13). One can verify that the family $P^{w}=\left(P_{t}^{w}\right)_{t \geq 0}$ forms a strongly continuous semigroup of contraction operators on $L^{2}(\Omega)$.

Definition 14. The semigroup $P^{w}=\left(P_{t}^{w}\right)_{t \geq 0}$ defined by (44) is called the $w$-Ornstein-Uhlenbeck semigroup on $L^{2}(\Omega)$.

We note that, with $w(k) \equiv 1$, the $w$-Ornstein-Uhlenbeck semigroup becomes the usual Ornstein-Uhlenbeck semigroup considered in $[2,14]$.

Theorem 15. Let $w: \mathbb{N} \rightarrow \mathbb{R}$ be a nonnegative function. Then the $w$-Ornstein-Uhlenbeck semigroup $P^{w}=\left(P_{t}^{w}\right)_{t \geq 0}$ is a Markov semigroup on $L^{2}(\Omega)$; namely, for all $t \geq 0$, it holds that

$$
0 \leq P_{t}^{w} \xi \leq 1 \quad \mathbb{P} \text {-a.e. }
$$

whenever $\xi \in L^{2}(\Omega)$ with $0 \leq \xi \leq 1 \mathbb{P}$-a.e.

Proof. Consider the operator $N_{w}$ in $L^{2}(\Omega)$ given by

$$
N_{w} \xi=\sum_{\sigma \in \Gamma} \#_{w}(\sigma)\left\langle Z_{\sigma}, \xi\right\rangle Z_{\sigma}, \quad \xi \in \operatorname{Dom} N_{w}
$$


where Dom $N_{w}$ denotes the domain of the operator $N_{w}$, which is defined as

$$
\begin{aligned}
& \operatorname{Dom} N_{w} \\
& =\left\{\left.\xi \in L^{2}(\Omega)\left|\sum_{\sigma \in \Gamma}\left[\#_{w}(\sigma)\right]^{2}\right|\left\langle Z_{\sigma}, \xi\right\rangle\right|^{2}<\infty\right\},
\end{aligned}
$$

where $\#_{w}(\cdot)$ is the $w$-counting measure defined by (13). It is easy to show that $N_{w}$ is a positive, self-adjoint, densely defined operator in $L^{2}(\Omega)$. And, moreover, by a direct calculation, we find that

$$
P_{t}^{w}=e^{-t N_{w}}, \quad t \geq 0,
$$

where $e^{-t N_{w}}$ means the spectral integral of the function $s \mapsto$ $e^{-t s}$ with respect to the spectral measure of $N_{w}$. Thus, $-N_{w}$ is exactly the infinitesimal generator of the semigroup $P^{w}=$ $\left(P_{t}^{w}\right)_{t \geq 0}$.

We now verify that $N_{w}$ and $\mathscr{E}_{w}$ share the following relationship:

$$
\mathscr{E}_{w}(\xi, \eta)=\left\langle\xi, N_{w} \eta\right\rangle, \quad \xi \in \mathrm{D}_{w}, \eta \in \operatorname{Dom} N_{w}
$$

with $\operatorname{Dom} N_{w} \subset \mathrm{D}_{w}$. In fact, if $\eta \in \operatorname{Dom} N_{w}$, then, by the well-known Cauchy inequality, we have

$$
\begin{aligned}
& \sum_{\sigma \in \Gamma} \#_{w}(\sigma)\left|\left\langle Z_{\sigma}, \eta\right\rangle\right|^{2} \\
& \quad \leq\left\{\sum_{\sigma \in \Gamma}\left[\#_{w}(\sigma)\right]^{2}\left|\left\langle Z_{\sigma}, \eta\right\rangle\right|^{2}\right\}^{1 / 2}\left\{\sum_{\sigma \in \Gamma}\left|\left\langle Z_{\sigma}, \eta\right\rangle\right|^{2}\right\}^{1 / 2} \\
& \quad<\infty
\end{aligned}
$$

which implies $\eta \in \mathrm{D}_{w}$. Thus, Dom $N_{w} \subset \mathrm{D}_{w}$. Let $\xi \in \mathrm{D}_{w}$ and $\eta \in \operatorname{Dom} N_{w}$. Then, by (17) and (21), we have

$$
\begin{aligned}
\mathscr{E}_{w}(\xi, \eta) & =\sum_{k=0}^{\infty} w(k)\left\langle\partial_{k} \xi, \partial_{k} \eta\right\rangle \\
& =\sum_{k=0}^{\infty} w(k) \sum_{\sigma \in \Gamma} \mathbf{1}_{\sigma}(k)\left\langle Z_{\sigma}, \xi\right\rangle\left\langle Z_{\sigma}, \eta\right\rangle \\
& =\sum_{\sigma \in \Gamma} \#_{w}(\sigma)\left\langle Z_{\sigma}, \xi\right\rangle\left\langle Z_{\sigma}, \eta\right\rangle .
\end{aligned}
$$

Here, we take use of the absolute convergence of the following series:

$$
\sum_{\sigma \in \Gamma} \sum_{k=0}^{\infty} \mathbf{1}_{\sigma}(k) w(k)\left\langle Z_{\sigma}, \xi\right\rangle\left\langle Z_{\sigma}, \eta\right\rangle,
$$

which can be verified straightforwardly. On the other hand, by the definition of $N_{w}$ and the expansion $\xi=\sum_{\sigma \in \Gamma}\left\langle Z_{\sigma}, \xi\right\rangle Z_{\sigma}$, we have

$$
\left\langle\xi, N_{w} \eta\right\rangle=\sum_{\sigma \in \Gamma} \#_{w}(\sigma)\left\langle Z_{\sigma}, \xi\right\rangle\left\langle Z_{\sigma}, \eta\right\rangle
$$

Thus, $\mathscr{E}_{w}(\xi, \eta)=\left\langle\xi, N_{w} \eta\right\rangle$.

Combining (48), (49), and Corollary 13 with the general theory of Dirichlet forms [8], we finally know that $P^{w}=$ $\left(P_{t}^{w}\right)_{t \geq 0}$ is a Markov semigroup.

\section{Discussion}

Let $X$ be a locally compact separable metric space and $\mu$ be a positive Radon measure on $X$. For a Dirichlet form $\mathscr{E}$ in $L^{2}(X, \mu)$, one thing researchers are interested in is whether it is regular [9]. Indeed, if $\mathscr{E}$ is a regular Dirichlet form in $L^{2}(X, \mu)$, then there is a symmetric Hunt process $M$ with $\mathscr{E}$ as its Dirichlet form. If, additionally, $\mathscr{E}$ possesses the local property, then the process $M$ is even a diffusion process [9]. In that case, the process $M$ is called the diffusion process associated with the Dirichlet form $\mathscr{E}$. It is well known that diffusion processes play an important role in many problems in mathematical physics.

As is shown, in our case, $\mathscr{E}_{w}$ is a Dirichlet form in $L^{2}(\Omega) \equiv$ $L^{2}(\Omega, \mathbb{P})$ for a positive function $w: \mathbb{N} \rightarrow \mathbb{R}$. On the other hand, being endowed with an appropriate metric, $\Omega$ can become a compact separable metric space and $\mathbb{P}$ can become a positive Radon measure on $\Omega$. It is then natural to consider whether or not $\mathscr{E}_{w}$ is regular and whether or not $\mathscr{E}_{w}$ possesses the local property. Such problems, however, are far from being simple and might be related to deeper topological properties of $\Omega$.

\section{Competing Interests}

The authors declare that they have no competing interests.

\section{Acknowledgments}

This work is supported by National Natural Science Foundation of China (Grant no. 11461061).

\section{References}

[1] C. Wang, H. Chai, and Y. Lu, "Discrete-time quantum Bernoulli noises," Journal of Mathematical Physics, vol. 51, no. 5, 2010.

[2] N. Privault, "Stochastic analysis of Bernoulli processes," Probability Surveys, vol. 5, pp. 435-483, 2008.

[3] I. Nourdin, G. Peccati, and G. Reinert, "Stein's method and stochastic analysis of Rademacher functionals," Electronic Journal of Probability, vol. 15, pp. no. 55, 1703-1742, 2010.

[4] C. Wang and J. Chen, "Quantum Markov semigroups constructed from quantum Bernoulli noises," Journal of Mathematical Physics, vol. 57, no. 2, Article ID 023502, 2016.

[5] A. M. Chebotarev and F. Fagnola, "Sufficient conditions for conservativity of minimal quantum dynamical semigroups," Journal of Functional Analysis, vol. 153, no. 2, pp. 382-404, 1998.

[6] P.-A. Meyer, Quantum Probability for Probabilists, vol. 1538 of Lecture Notes in Mathematics, Springer, Berlin, Germany, 1993.

[7] K. Parthasarathy, An Introduction to Quantum Stochastic Calculus, Springer Basel, Basel, Switzerland, 1992.

[8] S. Albeverio, "Theory of Dirichlet forms and applications," in Lectures on Probability Theory and Statistics, P. Bernard, Ed., vol. 1816 of Lecture Notes in Mathematics, pp. 1-106, Springer, Berlin, Germany, 2003.

[9] M. Fukushima, Y. Oshima, and M. Takeda, Dirichlet Forms and Symmetric Markov Processes, Walter De Gruyter, Berlin, Germany, 2011. 
[10] A. Beurling and J. Deny, "Dirichlet spaces," Proceedings of the National Academy of Sciences of the United States of America, vol. 45 , no. 2, pp. 208-215, 1959.

[11] S. Albeverio and R. Hoegh-Krohn, "Dirichlet forms and Markov semigroups on $\mathrm{C}^{*}$-algebras," Communications in Mathematical Physics, vol. 56, no. 2, pp. 173-187, 1977.

[12] T. Hida, J. Potthoff, and L. Streit, "Dirichlet forms and white noise analysis," Communications in Mathematical Physics, vol. 116, no. 2, pp. 235-245, 1988.

[13] C. Wang and J. Zhang, "Localization of quantum Bernoulli noises," Journal of Mathematical Physics, vol. 54, no. 10, Article ID 103502, 2013.

[14] C. Wang, Y. Lu, and H. Chai, "An alternative approach to Privault's discrete-time chaotic calculus," Journal of Mathematical Analysis and Applications, vol. 373, no. 2, pp. 643-654, 2011.

[15] M. Reed and B. Simon, Methods of Modern Mathematical Physics. I: Functional Analysis, Academic Press, San Diego, Calif, USA, 2nd edition, 1980. 


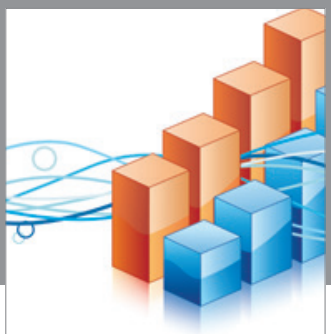

Advances in

Operations Research

vatem alat4

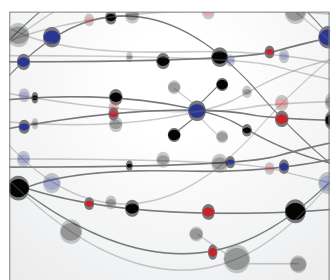

\section{The Scientific} World Journal
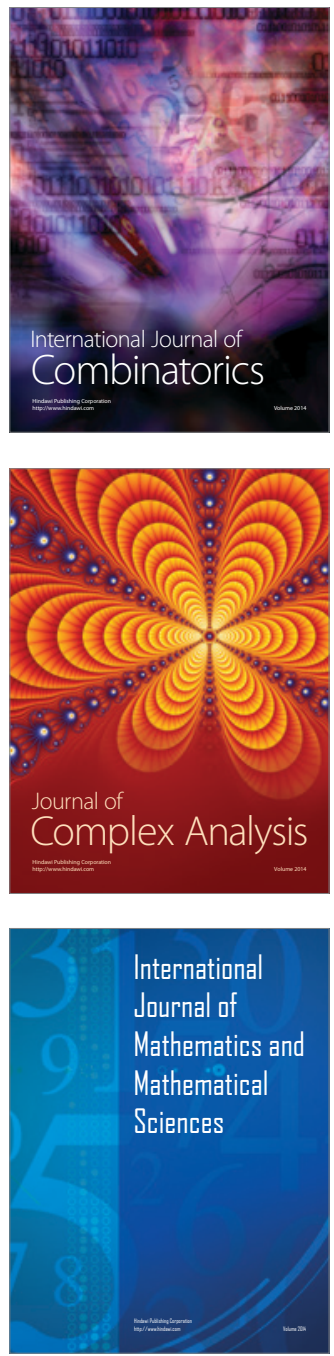
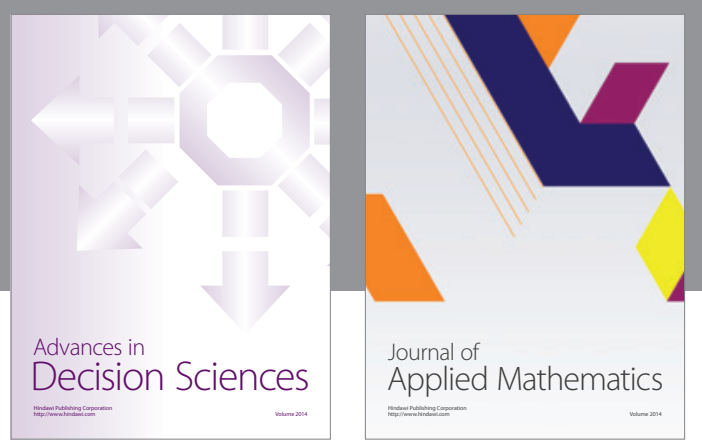

Algebra

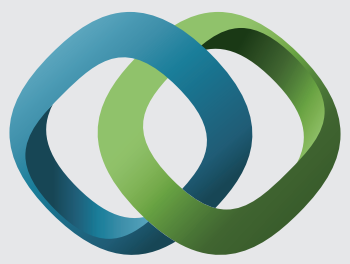

\section{Hindawi}

Submit your manuscripts at

https://www.hindawi.com
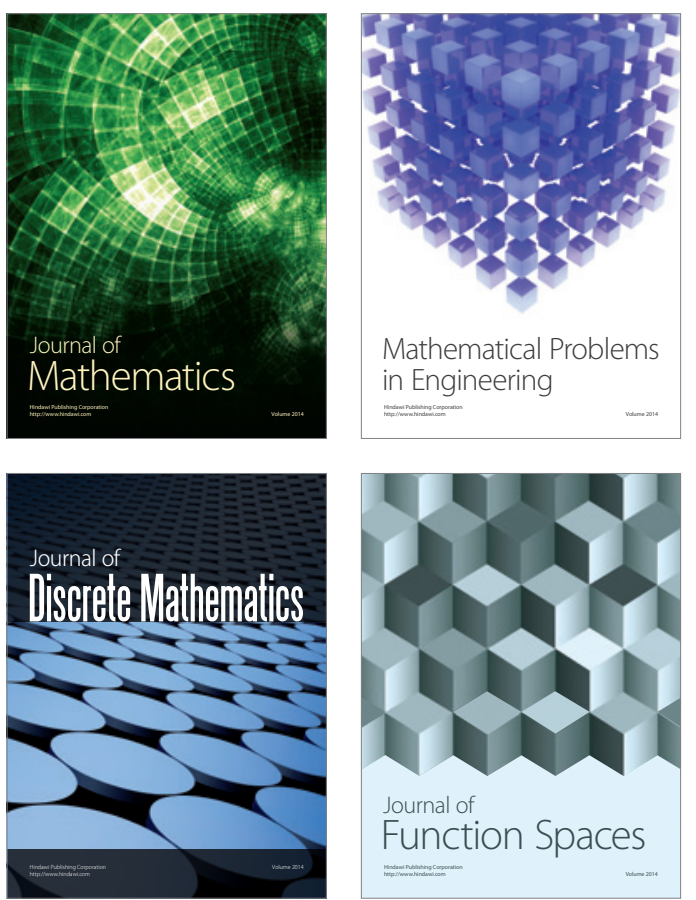

Mathematical Problems in Engineering
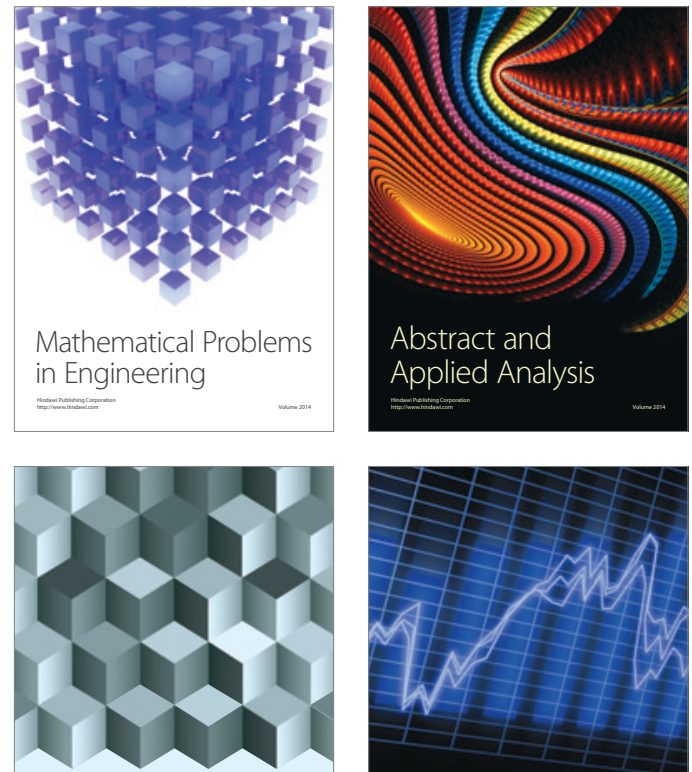

Journal of

Function Spaces

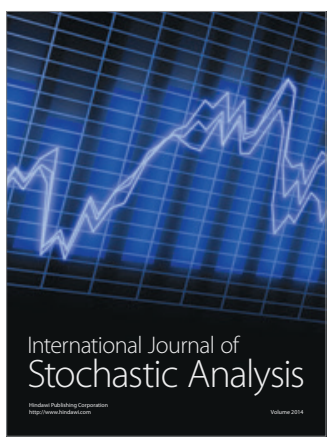

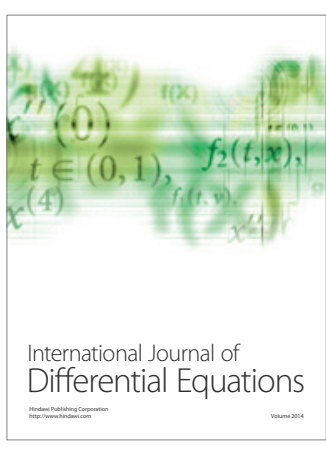
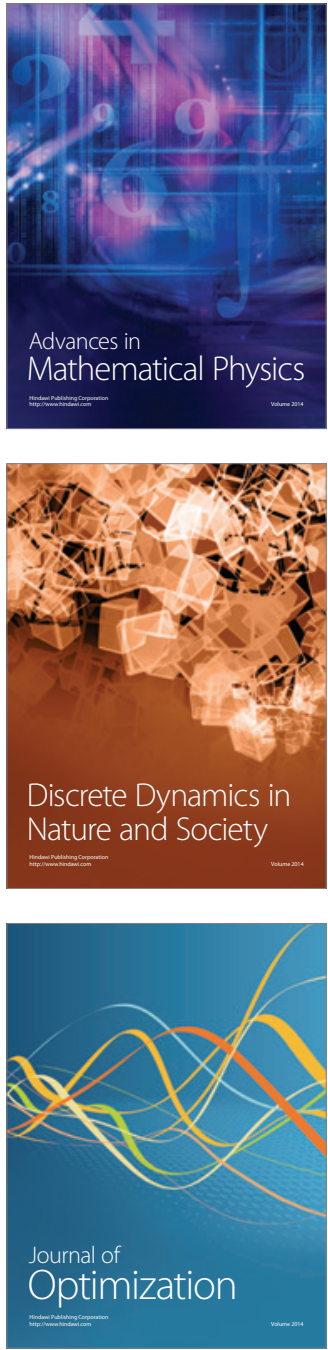